Geometry \& Topology

Volume 3 (1999) 211-233

Published: 14 July 1999

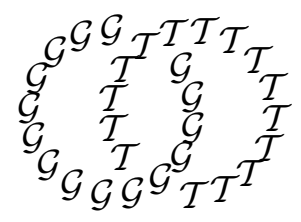

\title{
Lefschetz fibrations and the Hodge bundle
}

\author{
IVAN SMITH \\ New College, Oxford OX1 3BN, England \\ Email: smithi@maths.ox.ac.uk
}

\begin{abstract}
Integral symplectic 4-manifolds may be described in terms of Lefschetz fibrations. In this note we give a formula for the signature of any Lefschetz fibration in terms of the second cohomology of the moduli space of stable curves. As a consequence we see that the sphere in moduli space defined by any (not necessarily holomorphic) Lefschetz fibration has positive "symplectic volume"; it evaluates positively with the Kähler class. Some other applications of the signature formula and some more general results for genus two fibrations are discussed.
\end{abstract}

AMS Classification numbers Primary: 53C15

Secondary: 53C55, 58F99

Keywords: Symplectic geometry, Lefschetz fibration, stable curves, signature

Proposed: Ronald Stern

Seconded: Frances Kirwan, Walter Neumann
Received: 4 May 1999

Revised: 10 June 1999

Copyright Geometry and Topology 


\section{Statement of Results}

The second section contains an introduction to Lefschetz fibrations and motivation for the material of this paper, but we collect the main results here. Recall that all integral symplectic 4-manifolds admit Lefschetz fibrations which in turn are equivalent to isotopy classes of maps from a 2 -sphere to the moduli space of stable curves $\bar{M}_{g}$ (satisfying appropriate conditions). Once the genus is sufficiently large the isotopy class of the sphere becomes a symplectic invariant of the 4-manifold. If the 4-manifold is Kähler and the Lefschetz fibration is holomorphic the rational curve in $\bar{M}_{g}$ is a Kähler subvariety and in particular the Weil-Petersson form $\omega_{W P}$ is positive on the sphere.

1.1 Theorem For any symplectic Lefschetz fibration $f: X \rightarrow \mathbb{S}^{2}$ inducing $\phi_{f}: \mathbb{S}^{2} \rightarrow \bar{M}_{g}$ we have $\left\langle\left[\omega_{W P}\right],\left[\phi_{f}\left(\mathbb{S}^{2}\right)\right]\right\rangle>0$.

The statement has geometric content; the cohomological conditions on the sphere $\phi_{f}\left(\mathbb{S}^{2}\right)$ do not alone imply the result. The result is a consequence of the following more general result which extracts topological information on the 4 -manifold from the geometry of the sphere in moduli space.

1.2 Theorem With the notation as above, the signature of $X$ is given by

$$
\sigma(X)=\left\langle 4 c_{1}(\lambda),\left[\phi_{f}\left(\mathbb{S}^{2}\right)\right]\right\rangle-\delta
$$

where $\lambda \rightarrow \bar{M}_{g}$ denotes the Hodge bundle and $\delta$ is the number of critical fibres of the fibration.

A sketch of the proof can be found at the end of section two. This formula is a generalisation of one due to Atiyah for smooth fibrations, and related work by Meyer, Wolpert and others is well known ${ }^{1}$. In the algebraic case the theorem follows from standard Chern class equalities; nonetheless I have not seen the particular formula either applied or appear in the literature; and the extension to general symplectic fibrations (whilst unsurprising) seems new.

The signature theorem has various implications for the geometry of Lefschetz fibrations:

\subsection{Corollary}

- There are no Lefschetz fibrations with monodromy group contained in the Torelli group.

\footnotetext{
${ }^{1}$ The formula remains valid over an arbitrary base curve $B$ though in the sequel we shall usually leave the modifications to the reader.
} 
- Let $X \rightarrow \mathbb{P}^{1}$ be a genus two fibration with $n=10 m$ non-separating vanishing cycles and no others. Then $X$ is naturally a double cover of $\mathbb{S}_{\mathrm{sgn}(m)}$, where $\mathbb{S}_{\mathrm{sgn}(m)}$ denotes $\mathbb{S}^{2} \times \mathbb{S}^{2}$ if $m$ is even and the non-trivial sphere bundle over the sphere if $m$ is odd.

After earlier drafts of this work were distributed alternative proofs of the first corollary due to Li and Stipcisz also appeared. The author's proof also forms an Appendix to a preprint of Amoros, Bogomolov, Katzarkov and Pantev [1] who formulated the statement as a Conjecture. The existence of the double covers in the second corollary has been obtained, by different methods, by numerous authors but the identification of the base of the cover in terms of $m$ seems to have gone unnoticed. (The more general statement including reducible fibres is given in the text. We also give the classification of complex genus two Lefschetz fibrations without reducible fibres (5.5); this result, due to Chakiris, was rediscovered independently by the author, and to the best of his knowledge there is no published proof.)

Acknowledgements The material presented here is taken from [17]; I am grateful to my supervisor Simon Donaldson for conversations on these and related topics. Versions of most of this work have circulated informally and I apologise for the delays and duplications in its independent appearance.

\section{Recalling Lefschetz fibrations}

We begin with a lightning review of the concepts underlying the rest of the paper; a more leisurely tour of this material is taken in [17] amongst other sources. Recall that a Lefschetz fibration of a smooth 4-manifold $X$ comprises a surjection to $\mathbb{P}^{1}$, a submersion on the complement of finitely many points $p_{i}$ in distinct fibres, at which there are local complex coordinates (compatible with fixed global orientations on $X$ and $\mathbb{P}^{1}$ ) with respect to which the map takes the form $\left(z_{1}, z_{2}\right) \mapsto z_{1}^{2}+z_{2}^{2}$. We always assume that the fibres contain no $(-1)$-spheres ("relative minimality") so in particular the fibre genus is always strictly positive. The existence of a Lefschetz fibration guarantees that $X$ is a symplectic 4-manifold, whose topology is determined by a monodromy homomorphism

$$
\rho_{X}: \pi_{1}\left(\mathbb{P}^{1} \backslash\left\{f\left(p_{i}\right)\right\}\right) \rightarrow \Gamma_{g}
$$

where $\Gamma_{g}$ denotes the mapping class group of a closed oriented genus $g$ surface. The map $\rho_{X}$ maps the generators of the fundamental group which encircle a 
single critical point once in an anticlockwise fashion to positive Dehn twists in the mapping class group. These Dehn twists are about vanishing cycles; real circles in a fixed fibre which shrink along some given paths to the nodal points of the singular fibres. Thus the topology is completely encoded in an algebraic piece of data which is a word in such twists in the mapping class group, called a positive relation. We shall often refer to the values $\left\{f\left(p_{i}\right)\right\}$ - which are the critical values of $f$-by the set $\{\mathrm{Crit}\}$. The intrinsic symplectic form takes the shape $\omega=\tau+N f^{*} \omega_{\mathbb{S}}$ where $\tau$ is a closed form which is symplectic on the smooth fibres, and $\omega_{\mathbb{S}}$ is symplectic on the base $\mathbb{S}^{2} \cong \mathbb{P}^{1}$. The form is symplectic for sufficiently large $N$, and this "inflation" of the horizontal directions ensures that any local section (and hence multisection) of the fibration with suitable orientation can be made symplectic. The importance of the concept for us comes from

2.1 Theorem (Donaldson) Let $(X, \omega)$ be a symplectic 4-manifold for which $\omega$ is the lift of an integral class $[h]$. For sufficiently large integers $k$ the blow-up $X^{\prime}$ of $X$ at $k^{2}[h]^{2}$ distinct points admits a Lefschetz fibration over $\mathbb{P}^{1}$; each connected fibre, pushed back down to $X$, is Poincaré dual to $k[h]$.

The resulting Lefschetz fibration will be relatively minimal. We can always choose a compatible almost complex structure on $X^{\prime}$ such that

- the projection map to $\mathbb{P}^{1}$ is pseudoholomorphic;

- the structure is integrable in a sufficiently small tubular neighbourhood of each singular fibre.

Note that the exceptional sections are symplectic submanifolds of the blow-up $X^{\prime}$. The fibres of the fibration "downstairs" in $X$ before blowing-up form a Lefschetz pencil with finitely many base points. Once we have constructed a Lefschetz fibration on a symplectic manifold there is a natural symplectic form already given to us, without the existence result mentioned in the topological context above; the given form and the constructible form are deformation equivalent.

2.2 Remark Note that the canonical class of a symplectic manifold-which is uniquely defined - is independent of scalings of the symplectic form.

The choice of compatible almost complex structures or metrics with a fixed symplectic form on $X^{\prime}$ is contractible. Given one such choice, the smooth fibres of the fibration become metric, hence conformal and complex manifolds, that is Riemann surfaces of genus $g$. We therefore induce a map $\mathbb{P}^{1} \backslash\left\{f\left(p_{i}\right)\right\} \rightarrow M_{g}$ 
of a punctured sphere into the moduli space of curves. By the hypotheses of good local complex models, the singular fibres of the Lefschetz fibration are naturally stable curves (with a unique node in each) and the map completes to a map of the closed sphere into the Deligne-Mumford stable compactified moduli space $\bar{M}_{g}$. This map is then defined up to isotopy independent of the choice of metric or almost complex structure. The singular fibres correspond to the intersections of the sphere with the compactification divisor, and fall into two classes: irreducible fibres, where we collapse a non-separating cycle in the Riemann surface, and reducible fibres given by the one-point union of smooth Riemann surfaces of smaller genera. We shall see that the two kinds of singular fibre often play a somewhat different role in the sequel; each kind is counted by the intersection number of the sphere $\mathbb{P}^{1} \subset \bar{M}_{g}$ with the relevant components of the compactification divisor. Note also that the following four stipulations are geometrically equivalent:

- the local complex coordinates at the $p_{i}, f\left(p_{i}\right)$ all match with fixed global orientations;

- the monodromy homomorphism $\rho_{X}$ takes each of the obvious generators of the free group $\pi_{1}\left(\mathbb{P}^{1} \backslash\{\right.$ Crit $\left.\}\right)$ to a standard positive Dehn twist;

- the intersections of the sphere $\mathbb{P}^{1} \subset \bar{M}_{g}$ with the compactification divisor of stable curves are all locally positive;

- there is a symplectic structure on the total space $X^{\prime}$ which restricts on each smooth fibre to a symplectic form.

Note that from the point of view of the moduli space of curves, holomorphic spheres in $\bar{M}_{g}$ correspond to Kähler Lefschetz fibrations whilst smooth spheres give rise to more general symplectic 4-manifolds. One can make sense of Lefschetz fibrations over an arbitrary base curve $B$ and mutatis mutandis all the above comments apply.

A natural question to ask is how the algebraic topology of a 4-manifold is encoded in a Lefschetz description. By van Kampen's theorem it is easy to see that once a set of paths and vanishing cycles are chosen, the fundamental group of $X$ is just the quotient of $\pi_{1}$ (Fibre) by the classes generated by the vanishing cycles. This in turn gives the same description for the first homology group. In fact all the homology groups of $X$ are given by a pretty (short) chain complex essentially due to Lefschetz and presented in modern notation in [19] (see in particular Mumford's appendix to Chapter VI). To recall this, fix a set of paths in the base of the fibration and associated vanishing cycles $\delta_{i}$. We have

$$
0 \rightarrow H_{1}(F) \stackrel{\phi}{\longrightarrow} \mathbb{Z}^{r} \stackrel{\psi}{\longrightarrow} H_{1}(F) \rightarrow 0
$$


for $F$ a fixed smooth fibre and $r=\#\{$ Crit $\}$. We define the Picard-Lefschetz twist map $T_{j}$ by

$$
T_{j}(a)=a+\left\langle a, \delta_{j}\right\rangle \delta_{j}
$$

this is the effect of the Dehn twist about the cycle $\delta_{j}$ on the homology of the fibre $H_{1}(F)$, and $\langle\cdot\rangle$ denotes the intersection product. Because the composite of the monodromies in a Lefschetz fibration around a loop encircling all the critical values is trivial, we have the relation

$$
T_{r} T_{r-1} \cdots T_{1}=\text { id } .
$$

The maps in the sequence are defined by

$$
\begin{gathered}
\phi: u \mapsto\left(\left\langle T_{i-1} T_{i-2} \cdots T_{1} u, \delta_{i}\right\rangle\right)_{i} \\
\psi:\left(a_{i}\right) \mapsto \sum_{i} a_{i} \delta_{i} .
\end{gathered}
$$

The relation (2.3) ensures that the composite $\psi \circ \phi$ is zero and hence the sequence does indeed give a complex. The cokernel of $\psi$ is precisely the first homology group from the remarks above. Moreover the middle homology of the complex gives the group $G=H_{2}(X) /\langle[$ Fibre], [Section] $\rangle$. To see this, note that any element of $H_{2}(X)$ in the complement of the subspace spanned by fibres and sections projects to some graph in the base $\mathbb{S}^{2}$ whose endpoints are all critical values of the fibration; such an element is closed if and only if it arises from a union of vanishing discs bounding some homologically trivial cell in a fibre. Thus $G$ is indeed a quotient of ker $\psi$. Moreover, every 3-cell on $X$ defines on intersection with the fibres a 1-cell, and hence the third homology of $X$ can be computed by sweeping 1-cycles in fibres around the manifold via the monodromy maps. It follows - again recalling the relations given by (2.3) - both that the relations in $G$ are given by the image of $\phi$ whilst the group ker $\phi$ computes $H_{3}(X)$. Thus the word in vanishing cycles leads to an easy computation of the homology — both Betti numbers and torsion-for the manifold $X$.

Following this success, we ask for an expression for the signature of $X$. This is less straightforward. In principle one can compute the intersection matrix from the vanishing cycles via the sequence above, but the formulae are highly unmanageable. Ozbagci [15] has shown that using Wall's non-additivity formula - a souped up version of the Novikov additivity which gives the signature of a 4-manifold in terms of signatures of pieces resulting from cutting along a threemanifold - one can find an algorithm for computing signature from a word in vanishing cycles which can be fed to a computer. In this note we present a different formula which has the advantage of being elegant and in closed form 
but which has the disadvantage of starting not from a word in vanishing cycles but from a sphere in the moduli space $\bar{M}_{g}$. Nonetheless we shall see that the formula readily lends itself to certain applications to the topology and geometry of Lefschetz fibrations. Before giving the proof it will be helpful to assemble some facts on signature cocycles and on the moduli space of curves. The natural order in which to recall these does not really suit the proof of (1.2) and for the reader's convenience we give here the skeleton of the argument.

\section{A sketch of the proof}

(1) As with the Mayer-Vietoris sequence in homology, signature can be computed from the pieces of a decomposition of a manifold; we will cut a Lefschetz fibration into its smooth part and neighbourhoods of critical fibres.

(2) By the index theorem for manifolds with boundary, the signature of the smooth part can be expressed in terms of $\eta$-invariants of the boundary fibrations and the first Chern class of a determinant line bundle down the fibres. (This is precisely the Hodge bundle.)

(3) The determinant line bundle and $\eta$-invariant terms can be identified with a relative first Chern class of a topological line bundle defined by a signature cocycle in the group cohomology of the symplectic group. Thus the signature of the smooth part of the fibration is computed by a symmetric function on the symplectic group whose arguments are the monodromies around the boundary circles.

(4) By naturality properties of the cocycle and the above, the difference between evaluating the first Chern class of the Hodge bundle on a surface in $\bar{M}_{g}$ and the relative first Chern class on a surface with boundary in $M_{g}$ is entirely determined by the conjugacy classes of the monodromies in $S p_{2 g}(\mathbb{Z})$; since these are all equal for a Lefschetz fibration, the discrepancy is measured by a single integer for each genus.

(5) This integer is fixed by determining the theorem for at least one fibration at every genus; a Riemann-Roch theorem gives the theorem for all projective fibrations, completing the proof.

\section{The signature cocycle}

Much of the material here is drawn from Atiyah's pretty discussion in [3]. We start with Novikov's additivity formula, which states that if we decompose a 
4-manifold $X=X_{1} \cup_{Y} X_{2}$ along an embedded 3-manifold $Y$, then

$$
\sigma(X)=\sigma\left(X_{1}, Y\right)+\sigma\left(X_{2}, Y\right)
$$

where the left hand side denotes the signature of $X$ and for a manifold with boundary $(Z, \partial Z)$ the relative signature $\sigma(Z, \partial Z)$ denotes the difference between the number of positive and negative eigenvalues on the intersection form in the middle cohomology of $Z$ (but this form is no longer non-degenerate). Alternatively, on the image of the relative cohomology $H^{2}(Z, \partial Z) \hookrightarrow H^{2}(Z)$ the intersection form is non-degenerate and we take the signature of the form restricted to this subspace.

Let $X \rightarrow \mathbb{S}^{2}$ be a Lefschetz fibration. If we decompose $X$ into tubular neighbourhoods of the various singular fibres and a swiss cheese then by Novikov additivity we write the signature as the signature of the fibration over $\mathbb{S}^{2} \backslash\{$ Discs $\}$ corrected by the sums of the local signatures. An easy computation, noting that the total space of such a tube retracts to the singular fibre, gives that

- $\sigma(Z, \partial Z)=-1$ for a neighbourhood of a reducible singular fibre (separating vanishing cycle)

- $\sigma(Z, \partial Z)=0$ for a neighbourhood of an irreducible singular fibre (nonseparating vanishing cycle).

Thus we know $\sigma(X)=-s+\sigma(W, \partial W)$ where $s$ is the number of separating vanishing cycles and $W$ is the preimage $f^{-1}\left(\mathbb{S}^{2} \backslash D\right), D$ a neighbourhood of the set of critical values. Following [3] it is now natural to introduce the notation $\sigma\left(A_{1}, \ldots, A_{r}\right)$ for the signature of the 4 -manifold $Z$ which is a fibration by genus $g$ curves over a sphere with $r+1$ ordered open discs deleted, and for which the monodromies around the first $r$ boundary circles are given by elements $A_{i} \in \Gamma_{g}$. It follows of course that the monodromy about the last boundary circle is just the inverse of the ordered product of these matrices, for the fundamental group of the base is free. Write $\mathbb{S}^{2} \backslash D=\Sigma$. The topology of $Z$ is determined by a representation $\pi_{1}(\Sigma) \rightarrow \Gamma_{g}$ which may be composed with the standard representation $\Gamma_{g} \rightarrow S p_{2 g}(\mathbb{Z})$; this gives a flat vector bundle $E$ over $\Sigma$ with fibre the first complex cohomology of the fibre. On this vector bundle there is a non-degenerate skew-symmetric form given by the cup-product $\langle\cdot\rangle$ on $H^{1}$ (Fibre). Combining this skew form with the cup-product on classes from the base we obtain an indefinite Hermitian structure on the single vector space $H^{1}\left(\Sigma, \partial \Sigma ; H^{1}(\right.$ Fibre $\left.)\right)$ given by the cohomology with local coefficients in $E$.

3.1 Lemma [3] The signature $\sigma\left(A_{1}, \ldots, A_{r}\right)$ of $Z$ is the signature of the Hermitian form defined above on the cohomology group with local coefficients $H^{1}\left(\Sigma, \partial \Sigma ; H^{1}\right.$ (Fibre) $)$. 
The proof amounts to a careful application of the Leray-Serre spectral sequence for a fibration. That only the homologically non-trivial monodromies enter is unsurprising; signature after all is a homological invariant. It follows that the signature of a Lefschetz fibration is completely determined by the number of separating vanishing cycles $s$ and the value of the function $\sigma\left(\left\{A_{i}\right\}\right)$ where the $A_{i}$ can now be taken to be the symplectic matrices corresponding to the monodromies about homologically essential vanishing cycles. Applying the Novikov property to this symmetric function on the symplectic group gives the following critical relation:

$$
\sigma\left(A_{1}, A_{2}, A_{3}\right)=\sigma\left(A_{1}, A_{2}\right)+\sigma\left(A_{1} A_{2}, A_{3}\right)
$$

and hence, splitting the sphere with four holes in two distinct ways,

$$
\sigma\left(A_{1}, A_{2}\right)+\sigma\left(A_{1} A_{2}, A_{3}\right)=\sigma\left(A_{2}, A_{3}\right)+\sigma\left(A_{2} A_{3}, A_{1}\right) .
$$

This last formula is exactly the 2-cocycle condition in group cohomology, and it follows that we have defined an element $\left.[\sigma] \in H^{2}\left(S p_{2 g}(\mathbb{R}) ; \mathbb{Z}\right)\right)$. Such group cohomology elements correspond (cf Lemma (3.4)) to central extensions of the group by the integers and we have an associated sequence

$$
0 \rightarrow \mathbb{Z} \rightarrow S p^{\sigma} \rightarrow S p_{2 g}(\mathbb{R}) \rightarrow 0 ;
$$

moreover again by standard properties of group cohomology [7] there is a section to the last map $c^{\sigma}: S p_{2 g} \rightarrow S p^{\sigma}$ with the properties that

- the product $c^{\sigma}\left(A_{1}\right) c^{\sigma}\left(A_{2}\right) c^{\sigma}\left(A_{1} A_{2}\right)^{-1}$ gives a well-defined element of the central factor $\mathbb{Z}$ for any $A_{i} \in S p_{2 g}(\mathbb{R})$,

- $\sigma\left(A_{1}, A_{2}\right)=c^{\sigma}\left(A_{1}\right) c^{\sigma}\left(A_{2}\right) c^{\sigma}\left(A_{1} A_{2}\right)^{-1}$ for any $A_{i} \in S p_{2 g}(\mathbb{R})$.

The central point is that this section $c^{\sigma}$ has an interpretation in terms of a line bundle. Recall that the cohomology groups down the fibres of $Z \rightarrow \Sigma$ formed a flat vector bundle $E$ with a symplectic structure. If we complexify $E$ then the form $i\langle\cdot\rangle$ is Hermitian of type $(g, g)$ and we can choose a splitting $E_{\mathbb{C}}=E^{+} \oplus E^{-}$ into maximal positive definite and negative definite subspaces. Such splittings correspond to reducing the structure group of $E_{\mathbb{C}}$ from $U(g, g)$ to $U(g) \times U(g)$; the quotient homogeneous space is contractible so such splittings necessarily exist and are unique up to homotopy.

3.2 Lemma [3] Let $L$ be the line bundle $\left(\operatorname{det} E^{+}\right) \otimes\left(\operatorname{det} E^{-}\right)^{-1}$. The section $c^{\sigma}$ defines a homotopy class of trivialisations of $L^{2}$ over any loop $\gamma \subset \Sigma$ and hence a relative first Chern class $c_{1}\left(L^{2} ; c^{\sigma}\right) \in H^{2}(\Sigma, \partial \Sigma) \cong \mathbb{Z}$. Then working over a sphere with three discs deleted

$$
\sigma\left(A_{1}, A_{2}\right)=c^{\sigma}\left(A_{1}\right) c^{\sigma}\left(A_{2}\right) c^{\sigma}\left(A_{1} A_{2}\right)^{-1}=c_{1}\left(L^{2} ; c^{\sigma}\right)
$$


for any $A_{i} \in S p_{2 g}$.

Since we have Novikov additivity for $\sigma\left(\left\{A_{i}\right\}\right)$ and relative Chern classes behave well with respect to connected sums, it follows that for any fibration $Z \rightarrow \Sigma$ we have

$$
\sigma(Z, \partial Z)=\sigma\left(\left\{A_{i}\right\}\right)=c_{1}\left(L^{2} ; c^{\sigma}\right) .
$$

Therefore to understand the signature of a Lefschetz fibration we need only understand this relative first Chern class and its trivialisation over loops as defined by $c^{\sigma}$. Before turning to this we recall one well known observation which is relevant both above and in the sequel. The section $c^{\sigma}$ is in fact unique, since the difference between any two choices would give a homomorphism from the group $S p_{2 g}$ to the integers. But no such homomorphisms can exist; for there is a canonical homomorphism from the mapping class group $\Gamma_{g}$ onto $S p_{2 g}$ and

3.4 Lemma The mapping class group $\Gamma_{g}$ is perfect for $g \geq 3$ and has finite cyclic abelianisation for $g=1,2$.

This result is well known; $\left(\Gamma_{1}\right)_{a b}=\mathbb{Z}_{12}$ whilst $\left(\Gamma_{2}\right)_{a b}=\mathbb{Z}_{10}$. For $g \geq 3$ the usual proof simply writes a generating Dehn twist as an explicit product of commutators. More in line with our thinking is the following geometric sketch. It was shown by Wolpert [18] that for genus $g \geq 3$ the second cohomology of the moduli space of stable curves is generated by a Kähler class and the divisors given by the components of the compactifying locus of stable curves. By Poincaré duality it follows that there are homology elements $\left[C_{i}\right]$ which have algebraic intersection 1 with the $i$-th component of the compactification divisor and 0 with all others. We can represent these classes by embedded surfaces; since the moduli space is of high dimension (and the orbifold loci of high codimension) we can tube away excess intersections, at the cost of increasing genus, and even find representing surfaces $C_{i}$ with geometric intersection numbers with the stable divisor the same as the algebraic intersection numbers. But the fundamental group of a surface of genus $g$ with one boundary circle admits the presentation

$$
\left\langle a_{1}, b_{1}, \ldots, a_{g}, b_{g}, \partial \mid \prod\left[a_{i}, b_{i}\right]=\partial\right\rangle .
$$

Thus in the fibration of curves over the surface $C_{i}$ defined by the universal property of the moduli space, the monodromy about the unique singular fibrea standard positive Dehn twist - is expressed as a product of commutators. Since we can do this for any isotopy class of Dehn twist the result follows. 


\section{Hodge lines and determinant lines}

To warm up we will treat the case of a projective fibration $f: X \rightarrow B$ and introduce some of the objects that appear in the final statement of the main theorem. (In fact the proof of (1.2) will not crucially rely on a separate treatment for the projective case but some aspects are simplified by giving such an argument, and it gives some structure to the theory of the Hodge bundle we want to quote.) Now $d f$ has maximal rank only away from $\{$ Crit $\}$ the set of critical points $p_{1}, \ldots, p_{r}$ of $f$, but there is an exact sequence of sheaves

$$
0 \rightarrow f^{*} \Omega_{B}^{1,0} \rightarrow \Omega_{X}^{1,0} \rightarrow \Omega_{X / B}^{1} \rightarrow 0
$$

where the last term is defined by the sequence. Away from $\{$ Crit $\}$ there is an isomorphism $\Omega_{X / B}^{1} \cong \mathcal{K}_{X} \otimes f^{*} T_{B}^{1,0}$ of bundles.

4.1 Definition The line bundle $\omega_{X / B}=\mathcal{K}_{X} \otimes f^{*} T_{B}^{1,0}$ is called the dualising sheaf of $f$.

The adjunction formula says that if $C$ is a smooth curve in a complex surface $Z$ then $\mathcal{K}_{C}=\left.\mathcal{K}_{Z} \otimes \mathcal{O}_{Z}(C)\right|_{C}$. For singular $C$ we define the "dualising sheaf" for $C$ via $\omega_{C}=\left.\mathcal{K}_{Z} \otimes \mathcal{O}_{Z}(C)\right|_{C}$ where $\mathcal{O}_{Z}(C)$ is still the line bundle associated to the divisor $C$. Despite the definition this is naturally associated to $C$, independent of its embedding in any ambient surface. Since in a Lefschetz fibration all the fibres have trivial normal bundles, it follows that $\left.\omega_{X / B}\right|_{X_{b}}=\omega_{X_{b}}$ for each fibre $X_{b}=f^{-1}(b)$. The results of the following theorem are drawn from [5], Chapter III, sections 11 and 12 . Write $f_{*} \mathcal{F}=R^{0} f_{*} \mathcal{F}$.

4.2 Theorem Let the notation be as above; suppose $f$ has generic fibre genus $g$.

(1) $f_{*} \omega_{X / B}$ is locally free of rank $g$.

(2) $R^{1} f_{*} \omega_{X / B}$ is the trivial line bundle $\mathcal{O}_{B}$ (and the higher direct images vanish for reasons of dimension).

These facts require that $f$ have connected fibres. The following definition is formulated under the naive assumption that the universal curve $\mathcal{C} M_{g} \rightarrow M_{g}$ exists and extends to the compactification. Whilst this fails because of orbifold problems, the failure is in a real sense only technical; we could work instead with specific families and the "moduli functor" [2] if necessary.

4.3 Definition Write $\lambda_{B}=\wedge^{g} f_{*} \omega_{X / B}$, the Hodge bundle over $B$. If the fibration $f$ gives a map $\phi_{f}: B \rightarrow \bar{M}_{g}$ then $\lambda_{B}=\phi_{f}^{*} \lambda$ for $\lambda$ the Hodge bundle of the universal curve $\mathcal{C} \bar{M}_{g} \rightarrow \bar{M}_{g}$. 
The Hodge bundle is well known to generate the Picard group of line bundles on $M_{g}$ and to extend to $\bar{M}_{g}$. We have two cohomology classes $c_{1}(\lambda), \delta \in H^{2}\left(\bar{M}_{g}\right)$ where $\delta$ "counts intersections with the divisor of stable curves"; that is, $\delta$ is the first Chern class of the line bundle with divisor $\bar{M}_{g} \backslash M_{g}$, or the Poincaré dual of the fundamental class of $\bar{M}_{g} \backslash M_{g}$. For projective fibrations Mumford deduces the following from the Grothendieck-Riemann-Roch theorem [14]:

4.4 Proposition Let $f: X \rightarrow B$ be a complex Lefschetz fibration of a projective surface. Let [Crit] denote the cohomology class defined by the critical points of $f$ viewed as a subvariety of $X$. Then

$$
12 c_{1}\left(\lambda_{B}\right)=f_{*}\left(c_{1}^{2}\left(\omega_{X / B}\right)+[\text { Crit }]\right) .
$$

The geometry here enters in the form of an exact sequence

$$
0 \rightarrow \Omega_{X / B}^{1} \rightarrow \omega_{X / B} \rightarrow \omega_{X / B} \otimes \mathcal{O}_{\{\text {Crit }\}} \rightarrow 0 .
$$

For the structure sequence of $\{$ Crit $\} \subset X$ gives

$$
0 \rightarrow \mathcal{I}_{\{\text {Crit }\}} \rightarrow \mathcal{O}_{X} \rightarrow \mathcal{O}_{\{\text {Crit }\}} \rightarrow 0
$$

where $\mathcal{I}_{\{\text {Crit }\}}$ is the ideal sheaf of $\{$ Crit $\}$ in $X$. The result follows on tensoring with $\omega_{X / B}$ provided we show that $\mathcal{I}_{\{\text {Crit }\}} \otimes \omega_{X / B}=\Omega_{X / B}^{1}$. Now away from $\{$ Crit $\}$ the sequence

$$
0 \rightarrow f^{*} \Omega_{B}^{1,0} \rightarrow \Omega_{X}^{1,0} \rightarrow \Omega_{X / B}^{1} \rightarrow 0
$$

is an exact sequence of bundles and $\Omega_{X / B}^{1}, \omega_{X / B}$ coincide. Near a point of \{Crit\} $f$ has local form $\left(z_{1}, z_{2}\right) \mapsto z_{1} z_{2}$ for suitable coordinates. Accordingly

$$
\Omega_{X / B}^{1}=\operatorname{coker}\left(f^{*} \Omega_{B}^{1,0} \rightarrow \Omega_{X}^{1,0}\right)=\frac{\mathcal{O}_{X} d z_{1}+\mathcal{O}_{X} d z_{2}}{\mathcal{O}_{X} \cdot\left(z_{1} d z_{2}+z_{2} d z_{1}\right)}
$$

Now $\mathcal{I}_{\{\text {Crit\} }}$ is by definition the sheaf of ideals generated by $\left\langle z_{1}, z_{2}\right\rangle$ over $\mathcal{O}_{X}$, whilst the dualising sheaf $\omega_{X / B}=\mathcal{O}_{X} \cdot\left(d z_{1} \wedge d z_{2}\right) \otimes f^{*}(\partial / \partial t)$ for $t$ a coordinate on $B$. Explicitly

$$
f^{*} \frac{\partial}{\partial t}=\frac{1}{2 z_{2}} \frac{\partial}{\partial z_{1}}+\frac{1}{2 z_{1}} \frac{\partial}{\partial z_{2}} \Longrightarrow \omega_{X / B}=\mathcal{O}_{X} \cdot\left(\frac{d z_{2}}{z_{2}}-\frac{d z_{1}}{z_{1}}\right) .
$$

Now consider the transformation on $\mathcal{O}_{X} \cdot\left\langle d z_{1}, d z_{2}\right\rangle$ generated by

$$
d z_{1} \mapsto z_{1} \frac{d z_{2}}{z_{2}}-d z_{1} \quad ; \quad d z_{2} \mapsto z_{2} \frac{d z_{1}}{z_{1}}-d z_{2}
$$

and see the local forms for $\Omega_{X / B}^{1}$ and $\mathcal{I}_{\{\text {Crit }\}} \otimes \omega_{X / B}$ are clearly equal. 
The Grothendieck-Riemann-Roch theorem states that

$$
\operatorname{ch}\left(f_{!} \mathcal{F}\right)=f_{*}\left(\operatorname{ch} \mathcal{F} \cdot \mathcal{T}\left(T_{X / B}^{1}\right)\right) \quad[\mathrm{GRR}]
$$

for $\mathcal{F}$ any coherent sheaf on an irreducible non-singular projective variety, ch the Chern character and $\mathcal{T}$ the Todd class; recall also that $f_{!} \mathcal{F}=\sum(-1)^{i} R^{i} f_{*} \mathcal{F}$. The relative tangent sheaf $T_{X / B}^{1}$ of the map $f$ is defined via $T_{X / B}^{1}=T_{X}^{1,0}-$ $f^{*} T_{B}^{1,0}$ as an element of $K$-theory. The usual expansions of Chern characters and Todd classes, combined with taking total Chern classes in the exact sequence (4.5), give Mumford's result.

4.6 Corollary The main result (1.2) is valid for projective fibrations.

Proof By Hirzebruch's classical theorem we know

$$
\sigma(X)=\frac{\left\langle p_{1}(T X),[X]\right\rangle}{3} .
$$

Using $p_{1}(T X)=c_{1}^{2}\left(T_{X}^{1,0}\right)-2 c_{2}\left(T_{X}^{1,0}\right)$ and from the definition of $\omega_{X / B}$ it follows that

$$
\begin{gathered}
c_{1}\left(T_{X}^{1,0}\right)=f^{*} c_{1}\left(T_{B}^{1,0}\right)-c_{1}\left(\omega_{X / B}\right) \\
\Longrightarrow \quad c_{1}^{2}\left(\omega_{X / B}\right)=p_{1}(T X)+2 c_{2}\left(T_{X}^{1,0}\right)+2 f^{*} c_{1}\left(T_{B}^{1,0}\right) \cdot c_{1}\left(\omega_{X / B}\right) \\
\Longrightarrow \quad f_{*} c_{1}^{2}\left(\omega_{X / B}\right)=f_{*} p_{1}(T X)+2 f_{*} c_{2}\left(T_{X}^{1,0}\right)+2 c_{1}\left(T_{B}^{1,0}\right) \cdot f_{*} c_{1}\left(\omega_{X / B}\right) .
\end{gathered}
$$

Now by the remarks after (4.1), $\left.\omega_{X / B}\right|_{X_{b}}=\omega_{X_{b}}=\left.\mathcal{K}_{X}\right|_{X_{b}}$ and since the canonical divisor of a genus $g$ curve has degree $2 g-2$ it follows that

$$
f_{*} c_{1}\left(\omega_{X / B}\right)=(2 g-2)[B] \in H^{0}(B, \mathbb{Q}) .
$$

Moreover in the top dimension $f_{*}$ commutes with evaluation and recalling $\chi(X)=\chi(B) \chi(F)+\delta$ the result follows.

Projective fibrations exist with fibres of every genus $g>0$ so we have now proven the result for at least one fibration of each genus. We now turn to the general smooth case in the framework of differential geometry. The signature of any Riemannian manifold can be defined as the index of a differential operator $d+d^{*}: \Omega^{+} \rightarrow \Omega^{-}$, where $\Omega^{ \pm}$are the eigenspaces for an involution $\phi \mapsto i^{b} \phi$ (some suitable power $b=b(p)$ ) and $\phi \in \Omega^{p}$. On a complex Riemann surface $M$ this signature operator is equivalent to

$$
\bar{\partial}: \Omega^{0,0}(M) \oplus \Omega^{1,0}(M) \rightarrow \Omega^{0,1}(M) \oplus \Omega^{1,1}(M) .
$$

The determinant line of this operator has fibre (using Serre duality)

$$
\mathcal{L}_{\text {det }}=\left[\operatorname{det} H^{0}\left(M, \Omega^{1}\right)\right]^{-2}
$$


thus fibrewise there is a naturally defined isomorphism $\lambda \equiv \mathcal{L}_{\text {det }}^{-1 / 2}$.

Now in general a determinant line bundle admits a canonical metric and connexion [10], and the holonomy of the connexion is given by an expression of the shape $\exp (i \eta(\cdot))$; that is, the exponential of an $\eta$-invariant of the boundary manifold. Usually there is no canonical choice of logarithm for this holonomy. However, for the particular case of the signature operator, such a canonical logarithm does exist. The reason for this special behaviour is that the zeroeigenforms for the relevant differential operator give rise to harmonic forms, which by Hodge theory are governed by the topology of the manifold; thus the dimensions of the zero-eigenspaces cannot jump as for a general differential operator.

The upshot is a canonical trivialisation via $\eta$-invariants for the determinant line bundle $\mathcal{L}_{\text {det }}$ over any circle and hence the boundary of $\Sigma$. The index theorem for manifolds with boundary [4] has famously been used to give a formula for the signature in terms of $L$-polynomials with a boundary correction term defined via $\eta$-invariants. Comparing the terms of this expression to the universal expression for the first Chern form of a determinant line bundle gives the central identity (compare to (3.3)!)

$$
\sigma(Z, \partial Z)=-2 c_{1}\left(\mathcal{L}_{\mathrm{det}} ; \eta\right)
$$

where the notation refers to a relative Chern class defined with respect to the $\eta$-invariant trivialisations over $\partial \Sigma$.

With all this preamble we can forge the bridge between the two approaches. The determinant line bundle comprises a piece $\mathcal{L}^{\prime}$ corresponding to non-zero eigenvalues of the differential operator which is canonically trivialised (topologically though not as a unitary bundle) by Quillen's canonical determinantal section, which by construction is non-vanishing there. Thus as a topological bundle the determinant line bundle is isomorphic to the bundle given by taking only the zero-eigenvalue spaces (of harmonic forms in $\Omega^{ \pm}$in our case):

$$
\mathcal{L}_{\text {det }} \cong_{\mathrm{TOP}}\left(\operatorname{det} H^{-}\right) \otimes\left(\operatorname{det} H^{+}\right)^{-1} \text {. }
$$

4.9 Lemma The line bundle $\mathcal{L}_{\text {det }}$ is topologically the dual of the line bundle $L_{\sigma}$ defined from the signature cocycle. Moreover the trivialisations of $\mathcal{L}_{\text {det }}^{2}$ and $L_{\sigma}^{-2}$ (defined by $\eta$-invariants and $c^{\sigma}$ respectively) coincide.

Proof That the line bundles are dual amounts to an identification of the positive and negative harmonic forms of $\Omega^{ \pm} \cap H^{1}$ (Fibre $)_{\mathbb{C}}$ with the positive and 
negative eigenspaces for the Hermitian form $i\langle\cdot\rangle$; then compare the formulae defining $L$ in (3.2) and (4.8). But by definition the $\Omega^{ \pm}$are defined to be eigenspaces for an operator whose index is signature and the $E^{ \pm}$comprising $L$ are the definite subspaces for the Hermitian form arising from the symplectic signature pairing. That the trivialisations agree is a consequence of the lemma (3.4). For if the two trivialisations differed then their difference would define a map from the set of components of the boundary $\partial \Sigma$ to $\mathbb{Z}$ depending only on the particular monodromies associated to these components. Moreover since we know that there are identities $(3.3,4.7)$ for all fibrations $Z \rightarrow \Sigma$ we always know that

$$
\sum_{\gamma \in \pi_{0}(\partial \Sigma)} \eta_{\text {triv }}(\gamma)+c_{\text {triv }}^{\sigma}(\gamma)=0
$$

Since the values of the trivialisations on loops depend only and naturally on the monodromies, this difference map defines $N: \Gamma_{g} \rightarrow \mathbb{Z}$ which (taking the above relation for $\Sigma$ a sphere with three discs removed) is a homomorphism. But by (3.4) we know the mapping class groups admit no such non-trivial homomorphisms.

Assembling our various identifications we have proven

$$
\sigma(X)=-s+4\left\langle c_{1}(\lambda),[\Sigma]\right\rangle
$$

where we interpret the right hand side with respect to the $\eta$-trivialisation still (and recall $s$ is the number of separating vanishing cycles). To produce the final formula (1.2) we now need to understand the exact nature of the holonomy term for one of the Dehn twist monodromies in a Lefschetz fibration. Atiyah gives the precise formula which shows the sense in which the $\eta$-invariant gives a canonical logarithm for the holonomy of the Quillen connexion:

$$
\delta \log \operatorname{det} D_{\text {Quillen }}=-i \pi \eta-\frac{1}{2} \log _{\text {sign }}(\operatorname{Monod}(\mathscr{H})) ;
$$

here in the final term, which is an integer, $\mathscr{H}$ is the "topological determinant bundle" $\mathscr{H}=\left(\operatorname{det} H^{+}\right)^{-1} \otimes \operatorname{det} H^{-}$. The monodromy denotes the particular element of $S p_{2 g}(\mathbb{Z})$ corresponding to the fibration over a given boundary circle, and $\log _{\text {sign }}$ denotes a choice of logarithm for this monodromy given by the explicit signature cocycle we began with. The final answer can therefore be given by a direct computation with this cocycle. More simply, given the work at the start of the section and the naturality properties of the signature of manifolds (and hence the cocycle), we know that the answer depends only on the conjugacy class of the monodromy in the mapping class group. Since all our monodromies are positive Dehn twists about non-separating vanishing cycles, 
we are interested in a single integer for each genus $g$. This is then determined by the computation of the signature for a single Lefschetz fibration with genus $g$ fibres. But we already know the answer for projective fibrations: writing $n$ for the number of non-separating vanishing cycles,

$$
\sigma(X)=-s-n+\left\langle 4 c_{1}(\lambda),\left[\mathbb{P}^{1}\right]\right\rangle
$$

which is just as we require.

Note that from this point of view the singular fibres enter the formula from naturally different perspectives; the separating ones because they affect $H^{2}$ and invoke a local contribution to signature, the non-separating ones because they affect $H^{1}$ and hence the global monodromy which detects the extent to which the manifold is not homologically a product.

\section{Applications to genus two fibrations}

In the last sections we give some applications of the signature formula and digress into some of the topics we encounter.

5.1 Example The moduli space of genus two curves is in fact globally affine, and the ample divisor given by $f_{*} c_{1}^{2}(\omega)$, for $\omega$ the dualising sheaf of the universal curve, is empty on $M_{2}$ and a linear combination of boundary divisors on the compactification. Mumford [14] has calculated precisely that at genus two

$$
10 c_{1}(\lambda)=\delta_{0}+2 \delta_{1}
$$

where $\delta_{0}, \delta_{1}$ are respectively the irreducible components of $\bar{M}_{2} \backslash M_{2}$ corresponding to curves which are generically irreducible or a union of two elliptic curves respectively. From the signature formula, we therefore see that the signature for a genus two Lefschetz fibration is determined completely by the numbers of non-separating and separating vanishing cycles $n$ and $s$ :

$$
\sigma(X)=-\frac{3}{5} n-\frac{1}{5} s
$$

This "fractional signature formula" was first established by Matsumoto [13] by related but different means.

We can now compare the two different formulae for signatures of genus two fibrations to some effect. Recall that $\left(\Gamma_{2}\right)_{a b}=\mathbb{Z}_{10}$ and hence for any genus two fibration we have $10 \mid(n+2 s)$. 
5.3 Proposition Let $(X, f)$ be a genus two Lefschetz fibration with $n+2 s=$ $10 \mathrm{~m}$. Write $\mathbb{S}_{\mathrm{sgn}(m)}$ for the product $\mathbb{S}^{2} \times \mathbb{S}^{2}$ if $m$ is even and for the non-trivial sphere bundle over the sphere if $m$ is odd. Then

$$
X \# s \overline{\mathbb{C P}}^{2} \stackrel{2: 1}{\longrightarrow} \mathbb{S}_{\operatorname{sgn}(m)} \# 2 s \overline{\mathbb{C P}}^{2} \supset C ;
$$

the blow-up of $X$ at $s$ points admits a smooth double cover over the blow-up of the relevant sphere bundle over a sphere at $2 s$ points, ramified over a smooth surface $C$.

Equivalently, $X$ is given by blowing down $(-1)$-spheres in the fibres of a fibration arising from double covering a non-minimal rational surface over a curve $C$ which is the canonical resolution of singularities of a curve $C^{\prime} \subset \mathbb{S}_{\operatorname{sgn}(m)}$ containing $s$ infinitely close triple points. (Such a point is given by the singularity at the origin of $z_{1}^{3}+z_{2}^{6}=0$; the curve has 3 sheets meeting mutually tangentially at this point, and the sheets are separated by two successive blow-ups.) Thus if $X$ has no reducible fibres then it has $10 \mathrm{~m}$ singular fibres for some $m$, and $X$ double covers the sphere bundle with "parity" the same as the parity of $m$, branched over a smooth two-dimensional surface $C$. As a piece of notation, recall that $\mathbb{F}_{k}=\mathbb{P}(\mathcal{O} \oplus \mathcal{O}(k))$ denotes the unique complex (or symplectic) ruled surface with symplectic sections of self-intersection $\pm k$; moreover all projective bundles over $\mathbb{P}^{1}$ are the projectivisations of (not uniquely determined) vector bundles.

Proof The proof of the proposition is reasonably straightforward and versions due to Fuller [11] and Siebert-Tian [16] have now appeared (an independent proof was given in [17]). The idea is simple; on choosing a metric all the smooth fibres are hyperelliptic Riemann surfaces and admit natural double branched covers over spheres. These can be patched together smoothly except near separating singular fibres. The point is that the map fibrewise is given by sections of the canonical bundle on the fibre, and the nodal points in reducible fibres are base points of the canonical system on a stable curve; for smooth or stable irreducible curves the canonical linear system has no base points and the branch locus varies smoothly. Near reducible fibres we can assume the complex structure is integrable and graft in a local holomorphic model.

This argument (and those of Fuller and Siebert-Tian) gives the base of the double cover the structure of a sphere bundle over the sphere but does not identify it beyond that. The signature formulae allow one to do precisely this. The ruled surface $\mathbb{F}_{k}=\mathbb{P}(E)=\mathbb{P}(\mathcal{L} \otimes E)$ for suitable rank two $E$ and any line bundle $\mathcal{L}$. Since $c_{1}(E) \equiv c_{1}(E \otimes \mathcal{L}) \bmod (2)$ and $\mathbb{F}_{k} \cong_{\text {diffeo }} \mathbb{F}_{k+2}$, to find 
the diffeomorphism type of the base from a monodromy equation we need only understand the parity of the first Chern class of any suitable bundle $E$ above. Specifically, in the complex case the map $X \rightarrow \mathbb{S}$ is the map defined by the sheaf $\omega_{X / \mathbb{P}^{1}}$ and we know

$$
c_{1}\left(f_{*} \omega_{X / \mathbb{P}^{1}}\right)=c_{1}\left(\Lambda^{2} f_{*} \omega_{X / \mathbb{P}^{1}}\right)=c_{1}(\lambda) ;
$$

and more generally the sphere fibres of the ruled surface are the projectivisations of spaces of holomorphic sections of the canonical bundles down the fibres of $X$. Thus for any fibration $X$ we can take $\operatorname{det} E=\lambda$. From (5.2) and (1.2) we know

$$
4 c_{1}(\lambda)-n-s=-\frac{3}{5} n-\frac{1}{5} s .
$$

It follows that the parity of $10 c_{1}(\lambda)$ and the parity of $n+2 s=10 m$ coincide, and that is precisely what we require.

We remark for completeness that these branched coverings can be used to give a classification of complex genus two fibrations without reducible fibres. For in these cases the branch locus is a complex curve and such curves are determined to smooth isotopy by their connectivity and homology class. In the connected case, we can "canonically" choose such a curve as follows; write $s_{0}$ and $s_{\infty}$ for the homology classes of sections of a ruled surface $\mathbb{F}_{k}$ of square $\pm k$ respectively and $F$ for the homology class of a fibre. For a curve in a class $\left|r s_{0}+m F\right|$ choose $m$ fibres and $r$ sections $^{2}$ meeting in disjoint nodes, giving a curve of $r+m$ components. Now under a complex deformation of the fibration the branch locus will be perturbed to a neighbouring smooth complex curve. We can clearly arrange for all the fibres to lie over some small complex disc in the base $\mathbb{P}^{1}$ and for all the nodes from intersection points of sections to lie outside the preimage of this disc. The monodromy of the Lefschetz fibration arises entirely from a neighbourhood of the $m$ fibres in the branch locus and the other nodes, since these are the only places where we obtain singular fibres upstairs. Smoothing the nodal branch locus over suitably chosen disjoint discs in the base sequentially, we see that the Lefschetz fibrations arising from different branch loci coincide according to

$$
\begin{aligned}
\left|r s_{0}+(2 m) F\right|_{\mathbb{F}_{k}} & \sim\left|r s_{0}\right|_{\mathbb{F}_{k}} \#_{\text {fibre }}\left|r s_{0}+2 m F\right|_{\mathbb{F}_{0}} \\
& \sim\left|r s_{0}\right|_{\mathbb{F}_{k}} \#_{m} \text { fibres }\left|r s_{0}+2 F\right|_{\mathbb{F}_{0}} \\
& \sim \#_{i=1}^{k}\left|r s_{0}\right|_{\mathbb{F}_{1}}^{(i)} \#_{j=1}^{m}\left|r s_{0}+2 F\right|_{\mathbb{F}_{0}}^{(j)} .
\end{aligned}
$$

\footnotetext{
${ }^{2}$ For the covers to be genus two fibrations we will need $r=6$ since a genus two curve double covers a sphere over six points; for the double cover to exist the branch divisor, and hence $m$, will have to be even.
}

Geometry and Topology, Volume 3 (1999) 
Thus all of the fibrations arising from connected branch loci can be expressed as fibre sums of two basic pieces. We have not yet considered disconnected branch loci. Suppose then the branch locus is a curve in the class $\left|a s_{0}\right|+\left|b s_{\infty}\right|$ comprising two disjoint smooth components. From standard results on rational surfaces [12] it follows that $b=1$. In order for the class to contain six sections (and hence yield a genus two fibration) we are reduced to the case $\left|5 s_{0}\right|+\left|s_{\infty}\right|$ on $\mathbb{F}_{k}$. Moreover we can write $k=2 l$ to obtain an even divisor (as an element of the Picard group, necessary for the existence of the double cover). Again by standard results this class contains no smooth connected curve, and by the fibre summation trick, it is enough to understand the fibration when $l=1$; note that we can build a disconnected branch locus from only disconnected pieces, and all the pieces in a fibre sum decomposition will be of this special form $\left|5 s_{0}+s_{\infty}\right|$ in some $\mathbb{F}_{2 m}$.

We have reduced all complex genus two fibrations with no reducible fibres to fibre sums of three basic examples. These can be computed in a variety of ways and we obtain the following classification result (originally due to Chakiris [8] by similar methods and discovered independently if much later by the present author $\left.^{3}\right)$ :

5.5 Theorem (Chakiris) Assume that a genus two fibration has no reducible fibres and Kähler total space. Then it is a fibre sum of the shape $A^{m} B^{n}=1$ or $C^{p}=1$ where $m, n, p \in \mathbb{Z}_{\geq 0}$ and the basic words $A, B, C$ are given by:

$$
\begin{aligned}
A:\left(\delta_{1} \delta_{2} \delta_{3} \delta_{4} \delta_{5} \delta_{5} \delta_{4} \delta_{3} \delta_{2} \delta_{1}\right)^{2}=1 & \sim X \stackrel{2: 1}{\longrightarrow} \mathbb{F}_{0} \supset\left|6 s_{\infty}+2 F\right| \\
B:\left(\delta_{1} \delta_{2} \delta_{3} \delta_{4} \delta_{5}\right)^{6}=1 & \sim X \stackrel{2: 1}{\longrightarrow} \mathbb{F}_{1} \supset\left|6 s_{0}\right| \\
C:\left(\delta_{1} \delta_{2} \delta_{3} \delta_{4}\right)^{10}=1 & \sim X \stackrel{2: 1}{\longrightarrow} \mathbb{F}_{2} \supset\left|5 s_{0}\right| \amalg\left|s_{\infty}\right| .
\end{aligned}
$$

It follows that if a genus two Kähler Lefschetz fibration contains no separating vanishing cycles, then the total space is simply-connected. The notation $X \stackrel{d: 1}{\longrightarrow}$ $Y \supset B$ indicates that $X$ is a $d$-fold branched cover of $Y$ totally ramified along the locus $B$. The Dehn twists $\delta_{i}$ are about curves in the "standard position" on a Riemann surface (c.f. [6] for instance). Note also that for the second family of words $C^{p}=1$ the monodromy group of the fibration is not full; but to obtain an exhaustive list it is sufficient to take all fibre sums by the identity diffeomorphism. The word $B=1$ corresponds to the genus two pencil on a $K 3$ which double covers $\mathbb{P}^{2}$ branched over a sextic.

\footnotetext{
${ }^{3}$ Chakiris' result has appeared in numerous articles but never accompanied by any kind of proof; his original work, in part being more ambitious, is somewhat dense. It therefore seems reasonable to present a version of the argument here.
} 


\section{$6 \quad$ Further applications}

At higher genera the moduli spaces are not affine, and signature is not in general determined by the combinatorial equivalence class of the fibration. However, for fibrations by hyperelliptic curves the statement is much easier. Recall that in the $(3 g-3)$-dimensional moduli space $M_{g}$ there is a distinguished $(2 g-1)$ dimensional locus of hyperelliptic curves, which forms a complex analytic subvariety $\mathscr{H}_{g}$. We can take the closure of this subvariety in $\bar{M}_{g}$. The cohomology classes $c_{1}(\lambda), \delta_{0}, \ldots, \delta_{[g / 2]}$ form a basis for $H^{2}\left(\bar{M}_{g}\right)$; here $\delta_{0}$ is the irreducible component of $\bar{M}_{g} \backslash M_{g}$ corresponding to generically irreducible curves, and the $\delta_{i}$ are the components corresponding to curves which generically have one component of genus $i$ and the other of genus $g-i$. We can restrict these classes to $\overline{\mathscr{H}}_{g}$ where we denote them by the same symbols. The following relation seems to be new:

6.1 Lemma For any hyperelliptic Lefschetz fibration there is an inequality

$$
(8 g+4) c_{1}(\lambda) \geq g \delta_{0}+\sum_{h=1}^{[g / 2]} 4 h(g-h) \delta_{h} .
$$

which is an equality when the base is a two-sphere.

But this follows immediately from (1.2) and a fractional signature formula (generalising the one for genus two above) which has been given by Endo [9].

As a more significant application we provide the answer to a conjecture of Amoros, Bogomolov, Katzarkov and Pantev [1]. The monodromy group of a Lefschetz fibration is the subgroup of the mapping class group generated by the Dehn twists about the vanishing cycles; that is, the image of the representation $\pi_{1}\left(\mathbb{P}^{1} \backslash\{\right.$ Crit $\left.\}\right) \rightarrow \Gamma_{g}$. Recall that the Torelli group is the subgroup of the mapping class group comprising elements which act trivially on homology; thus the monodromy group of $X \rightarrow \mathbb{P}^{1}$ is contained in the Torelli group if and only if all the vanishing cycles are separating. (We will sometimes refer to such a fibration as a "Torelli fibration".)

For Kähler fibrations this can clearly never happen; here is one classical proof. By taking the Jacobians down the fibres, a Kähler fibration gives a map from the smooth locus $\mathbb{P}^{1} \backslash\{$ Crit $\}$ to the moduli space of principally polarised abelian varieties $\mathcal{A}_{g}$. This map is holomorphic, and if the monodromy is trivial on homology groups and hence Jacobians, we can canonically complete to a holomorphic map of the closed sphere into $\mathcal{A}_{g}$. But this is possible only if the map 
is constant, for $\mathcal{A}_{g}$ is well known to be a bounded complex domain, and in particular contains no non-trivial holomorphic spheres.

In [1] it is conjectured ${ }^{4}$ that in fact there can be no fibrations with monodromy group contained in the Torelli group even in the absence of the Kähler assumption (used via holomorphicity of the map to $\mathcal{A}_{g}$ above). Indeed this is true:

6.2 Theorem There are no Lefschetz fibrations with monodromy group a subgroup of the Torelli group.

Proof Suppose such an $(X, f)$ exists; we work by contradiction. We mentioned before that another approach to signature uses Wall's non-additivity; an easy case of this approach [15] shows that if a fibration has $\delta$ separating vanishing cycles and no others, then its signature is given by $-\delta$. Comparing to the formula (1.2) this forces $\left\langle c_{1}(\lambda),\left[\mathbb{S}^{2}\right]\right\rangle=0$. We will prove that this quantity is positive.

We have $c_{2}(X)=4-4 g+\delta=2-2 b_{1}(X)+b_{2}(X)$ for any Lefschetz fibration with $\delta$ singular fibres; in our case, since all the vanishing cycles are homologically trivial, $b_{1}(X)=2 g$ and hence $b_{2}(X)-2=\delta$. Now the number of disjoint exceptional $(-1)$-spheres in $X$ is bounded by $b_{2}(X)$ since each contributes a new homology class. The fibre of the fibration has self-intersection zero; fibresumming $X$ with itself by the identity if necessary, we can see that if any fibration with monodromy group contained in the Torelli group exists, then one exists for which a section also has self-intersection even and in particular is not exceptional. Thus the number of $(-1)$-spheres in $X$ may be assumed to be bounded above by $b_{2}(X)-2=\delta$.

We invoke a powerful theorem of Liu: for any minimal symplectic 4-manifold $Z$ which is not irrational ruled, $c_{1}^{2}(Z) \geq 0$. In particular, blowing down all $(-1)$-spheres in $X$, we see that

$$
c_{1}^{2}(X)=c_{1}^{2}\left(X_{\min }\right)-\#\{(-1)-\text { spheres }\} \geq-\delta
$$

and hence

$$
c_{1}^{2}(X)+c_{2}(X) \geq 4-4 g
$$

provided $X$ is not irrational ruled. But we also know

$$
\frac{c_{1}^{2}(X)+c_{2}(X)}{12}=\frac{\sigma(X)+\chi(X)}{4}=1-g+\left\langle c_{1}(\lambda),\left[\mathbb{S}_{X}^{2}\right]\right\rangle .
$$

\footnotetext{
${ }^{4}$ The conjecture appeared in an early draft of the paper; the proof given here was incorporated as an Appendix to a later draft at the request of the authors.
} 
Rearranging this gives $\left\langle c_{1}(\lambda),\left[\mathbb{S}^{2}\right]\right\rangle>0$ which is the required contradiction. That leaves only the case of $X$ irrational ruled, but simple topological conditions show that such a manifold can have no Torelli fibration. For any Torelli Lefschetz fibration of $X=\Sigma_{h} \tilde{\times} \mathbb{S}^{2}$ has fibres of genus $h$ by considering $H_{1}(X)$, and then looking at $\pi_{1}(X)$ we see that in fact all the vanishing cycles must be nullhomotopic and hence $X$ was a trivial product.

There are fibrations over the torus with no critical fibres which have monodromy group contained in the Torelli group; just take $\mathbb{S}^{1} \times Y$ for $Y$ the mapping torus of a Dehn twist about a separating curve. The Lefschetz fibrations of these manifolds, however, cannot be Torelli.

6.3 Corollary For any Lefschetz fibration $X, \sigma(X)+\delta>0$. Moreover the sphere $\mathbb{S}_{X}^{2} \subset \bar{M}_{g}$ defined to isotopy by $X$ has positive "symplectic volume"; the symplectic Weil-Petersson form on $\bar{M}_{g}$ evaluates positively on $\mathbb{S}_{X}^{2}$.

The corollary again follows by comparing to Ozbagci's work; for he proves that each critical fibre changes the signature by $0, \pm 1$ as you add handles to a trivial bundle over the disc. Since we now know there must be a non-separating vanishing cycle, and his construction allows us to add that handle first and change the signature by a non-negative amount, we obtain the first result. In particular, it follows that for any smooth sphere in $\bar{M}_{g}$ with correct geometric intersection behaviour at the compactification divisors, the Chern class $c_{1}(\lambda)$ evaluates positively. The Kähler Weil-Petersson form is not quite a positive rational multiple of this Chern class and the boundary divisors; rather we have the identity (technically on the moduli stack)

$$
\frac{1}{2 \pi^{2}} \omega_{W P}=12 c_{1}(\lambda)-\delta
$$

An easy computation, however, shows that $c_{1}(\lambda)>0$ and $c_{1}^{2}(X)+8(g-1)>$ 0 together imply that the Weil-Petersson form evaluates positively. This is made more interesting by the following remark. Wolpert has computed the intersection ring of $\bar{M}_{g}$ and it follows from his computation that this is not a purely homological statement [18]; there are two dimensional homology classes which have positive intersections with all the components of $\bar{M}_{g} \backslash M_{g}$ but not with $c_{1}(\lambda)$. Thus the corollary is a kind of "symplectic ampleness" phenomenon, reliant on the local positivity of intersections with the stable locus. 


\section{References}

[1] J Amoros et al, Symplectic Lefschetz fibrations with arbitrary fundamental groups, preprint (1998) with an Appendix by Ivan Smith

[2] E Arabello, M Cornalba, The Picard groups of the moduli spaces of curves, Topology 26 (1987) 153-71

[3] M Atiyah, The logarithm of the Dedekind $\eta$-function, Math. Ann. 278 (1987) 335-380

[4] M Atiyah et al, Spectral asymmetry and Riemannian geometry I, Math. Proc. Camb. Phil. Soc. 77 (1975) 43-69

[5] W Barth, C Peters, A Van de Ven, Compact complex surfaces, Springer (1984)

[6] J Birman, Braids, links and mapping class groups, Princeton University Press (1975)

[7] K S Brown, Cohomology of groups, Springer (1982)

[8] K N Chakiris, The monodromy of genus two pencils, $\mathrm{PhD}$ thesis Colombia University (1978)

[9] H Endo, Meyer's signature cocycle and hyperelliptic fibrations, preprint (1998)

[10] D Freed, On determinant line bundles, from: "Mathematical Aspects of String Theory I", S T Yau (editor), World Scientific (1987)

[11] T Fuller, Genus two Lefschetz fibrations, preprint (1997)

[12] R Hartshorne, Algebraic geometry, Springer (1977)

[13] Y Matsumoto, Lefschetz fibrations of genus two - a topological approach, from: "The 37th Taniguchi Symposium on topology and Teichmüller spaces", S Kojima et al (editors), World Scientific (1996)

[14] D Mumford, Towards an enumerative geometry of the moduli space of curves, from: "Arithmetic and Geometry", A Artin and J Tate (editors), vol II Birkhäuser (1983)

[15] B Ozbagci, Signatures of Lefschetz fibrations, preprint (1998)

[16] B Siebert, G Tian, On hyperelliptic $C^{\infty}$-Lefschetz fibrations of 4-manifolds, preprint (1999)

[17] I Smith, Symplectic geometry of Lefschetz fibrations, Ph D thesis, Oxford University (1998)

[18] S Wolpert, On the homology of the moduli space of stable curves, Ann. of Math. 118 (1983) 491-523

[19] S Zariski, Algebraic surfaces, 2nd ed. Springer (1977) with appendices by D Mumford 